\title{
Teaching Video NeuroImages: Pathologic yawning
}

\section{A sign of brainstem involvement in acute disseminated encephalomyelitis?}

Veronica Birca, MD, Christine Saint-Martin, MD, and Kenneth A. Myers, MD, PhD

Neurology ${ }^{\circledR}$ 2020;94:e2497-e2498. doi:10.1212/WNL.0000000000009595

\section{Correspondence}

Dr. Myers

kenneth.myers@mcgill.ca

Figure Brain MRI

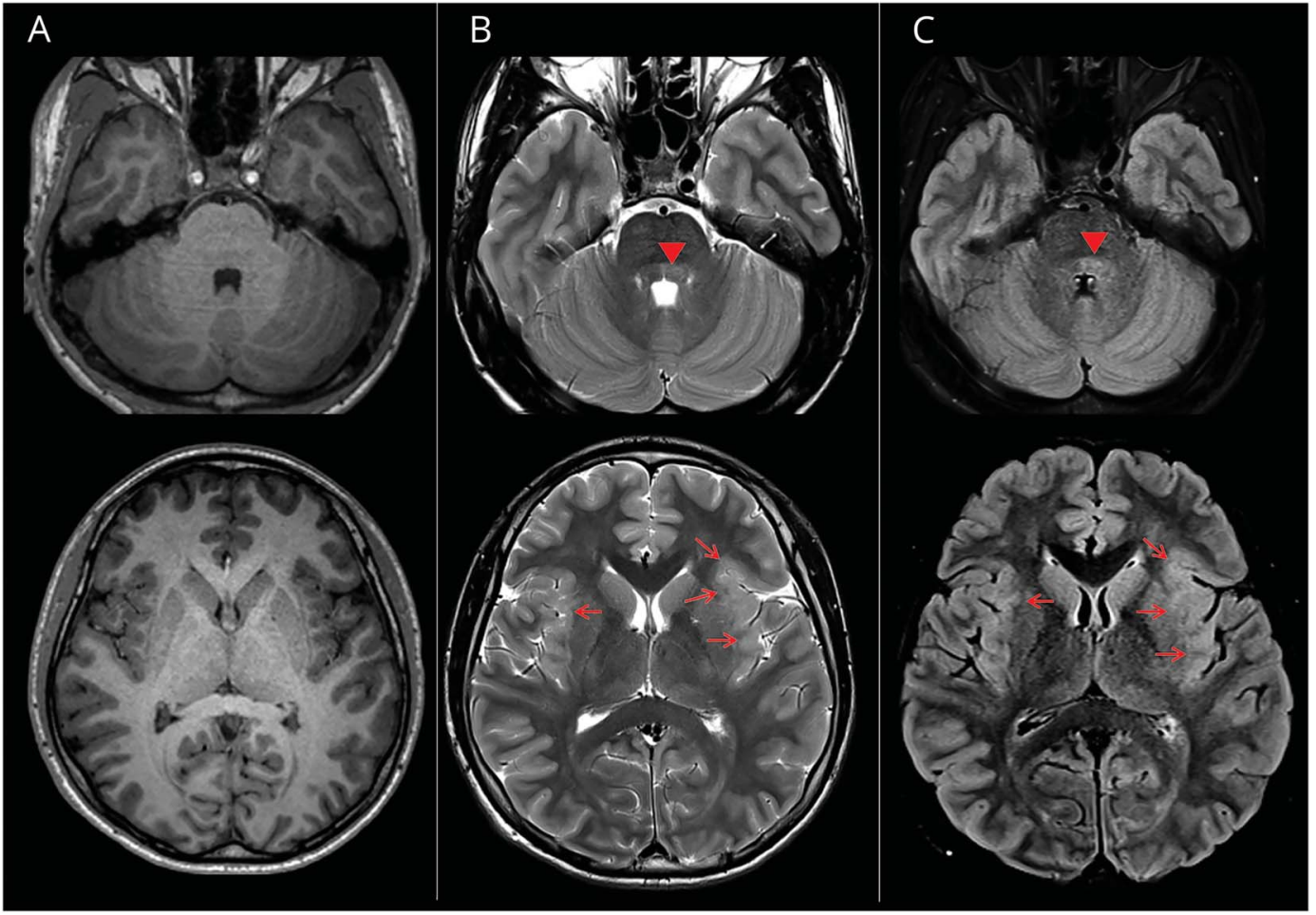

Axial views show normal T1 (A), asymmetric T2 (B), and fluid-attenuated inversion recovery (FLAIR) (C) hyperintensity of the dorsal brainstem involving the reticular formation (arrowheads) at the level of cranial nerves V, VI, and VII nuclei, and T2 and FLAIR hyperintensity of the bilateral, left more than right, insula (arrows).

A 15-year-old boy had acute-onset encephalopathy following a viral prodrome, associated with frequent, dramatic yawning without EEG change (video). There were no focal deficits on examination. CSF protein elevation $(1.72 \mathrm{~g} / \mathrm{L}$ ) without pleocytosis, brain MRI (figure), and significant improvement following methylprednisolone were consistent with acute disseminated encephalomyelitis (ADEM). Pathologic yawning is described in other demyelinating diseases, neuromyelitis optica spectrum disorder and multiple sclerosis, but is rarely reported with ADEM. Although prior reports postulated that brainstem and hypothalamic lesions are responsible, ${ }^{1}$ yawning is a complex reflex mediated by both supratentorial and infratentorial structures; its precise localization is not yet defined. ${ }^{2}$

\section{MORE ONLINE}

- Video

$\rightarrow$ Teaching slides

links.lww.com/WNL/

B96. 


\section{Study funding}

No targeted funding reported.

\section{Disclosure}

V. Birca and C. Saint-Martin report no relevant disclosures. K.A. Myers receives/has received research funding from the Research Institute of the McGill University Health Center, Citizens United for Research in Epilepsy, Savoy Foundation, and Dravet Canada. Go to Neurology.org/ $\mathrm{N}$ for full disclosures.

\section{Appendix Authors}

\begin{tabular}{|c|c|c|}
\hline Name & Location & Contribution \\
\hline $\begin{array}{l}\text { Veronica } \\
\text { Birca, MD }\end{array}$ & $\begin{array}{l}\text { Department of Pediatrics, } \\
\text { Division of Child Neurology, } \\
\text { Montreal Children's Hospital, } \\
\text { McGill University Health } \\
\text { Centre, Canada }\end{array}$ & $\begin{array}{l}\text { Conception and design; } \\
\text { acquisition, analysis, and } \\
\text { interpretation of data; } \\
\text { literature review; drafting } \\
\text { of manuscript and critical } \\
\text { revision; final approval }\end{array}$ \\
\hline
\end{tabular}

Appendix (continued)

\begin{tabular}{|c|c|c|}
\hline Name & Location & Contribution \\
\hline $\begin{array}{l}\text { Christine } \\
\text { Saint- } \\
\text { Martin, } \\
\text { MD }\end{array}$ & $\begin{array}{l}\text { Department of Medical } \\
\text { Imaging, Montreal Children's } \\
\text { Hospital, McGill University } \\
\text { Health Centre, Canada }\end{array}$ & $\begin{array}{l}\text { Acquisition, analysis, and } \\
\text { interpretation of data; } \\
\text { literature review; drafting } \\
\text { of manuscript and critical } \\
\text { revision; final approval }\end{array}$ \\
\hline $\begin{array}{l}\text { Kenneth A. } \\
\text { Myers, } \\
\text { MD, PhD }\end{array}$ & $\begin{array}{l}\text { Research Institute, } \\
\text { Department of Pediatrics, } \\
\text { Division of Child Neurology, } \\
\text { Montreal Children's Hospital, } \\
\text { McGill University Health } \\
\text { Centre, Canada }\end{array}$ & $\begin{array}{l}\text { Conception and design; } \\
\text { acquisition, analysis, and } \\
\text { interpretation of data; } \\
\text { literature review; drafting } \\
\text { of manuscript and critical } \\
\text { revision; supervision; final } \\
\text { approval }\end{array}$ \\
\hline
\end{tabular}

\section{References}

1. Lana-Peixoto MA, Callegaro D, Talim N, et al. Pathologic yawning in neuromyelitis optica spectrum disorders. Mult Scler Relat Disord 2014;3:527-532.

2. Krestel H, Bassetti CL, Walusinski O. Yawning: its anatomy, chemistry, role, and pathological considerations. Prog Neurobiol 2018;161:61-78. 


\section{Neurology}

Teaching Video NeuroImages: Pathologic yawning: A sign of brainstem involvement in acute disseminated encephalomyelitis?

Veronica Birca, Christine Saint-Martin and Kenneth A. Myers

Neurology 2020;94;e2497-e2498 Published Online before print May 4, 2020

DOI 10.1212/WNL.0000000000009595

This information is current as of May 4, 2020

\section{Updated Information \&} Services

References

Citations

Subspecialty Collections

Permissions \& Licensing

Reprints including high resolution figures, can be found at: http://n.neurology.org/content/94/23/e2497.full

This article cites 2 articles, 0 of which you can access for free at: http://n.neurology.org/content/94/23/e2497.full\#ref-list-1

This article has been cited by 2 HighWire-hosted articles: http://n.neurology.org/content/94/23/e2497.full\#\#otherarticles

This article, along with others on similar topics, appears in the following collection(s):

Acute disseminated encephalomyelitis

http://n.neurology.org/cgi/collection/acute_disseminated_encephalomy elitis

All Demyelinating disease (CNS)

http://n.neurology.org/cgi/collection/all_demyelinating_disease_cns All Pediatric

http://n.neurology.org/cgi/collection/all_pediatric

MRI

http://n.neurology.org/cgi/collection/mri

Information about reproducing this article in parts (figures,tables) or in its entirety can be found online at:

http://www.neurology.org/about/about_the_journal\#permissions

Information about ordering reprints can be found online:

http://n.neurology.org/subscribers/advertise

Neurology ${ }^{\circledR}$ is the official journal of the American Academy of Neurology. Published continuously since 1951, it is now a weekly with 48 issues per year. Copyright (C 2020 American Academy of Neurology. All rights reserved. Print ISSN: 0028-3878. Online ISSN: 1526-632X.

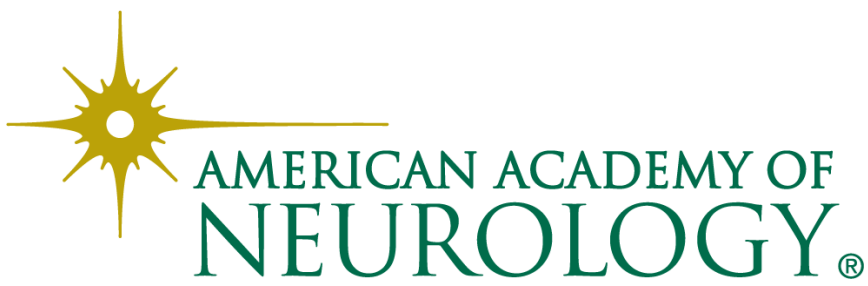

